\title{
Metadevices for the confinement of sound and broadband double-negativity behavior
}

\author{
Christensen, Johan; Liang, Z.; Willatzen, Morten
}

Published in:

Physical Review B Condensed Matter

Link to article, DOI:

10.1103/PhysRevB.88.100301

Publication date:

2013

Document Version

Publisher's PDF, also known as Version of record

Link back to DTU Orbit

Citation (APA):

Christensen, J., Liang, Z., \& Willatzen, M. (2013). Metadevices for the confinement of sound and broadband double-negativity behavior. Physical Review B Condensed Matter, 88(10), [100301].

https://doi.org/10.1103/PhysRevB.88.100301

\section{General rights}

Copyright and moral rights for the publications made accessible in the public portal are retained by the authors and/or other copyright owners and it is a condition of accessing publications that users recognise and abide by the legal requirements associated with these rights.

- Users may download and print one copy of any publication from the public portal for the purpose of private study or research.

- You may not further distribute the material or use it for any profit-making activity or commercial gain

- You may freely distribute the URL identifying the publication in the public portal

If you believe that this document breaches copyright please contact us providing details, and we will remove access to the work immediately and investigate your claim. 


\title{
Metadevices for the confinement of sound and broadband double-negativity behavior
}

\author{
J. Christensen, ${ }^{1,2,{ }^{*}}$ Z. Liang, ${ }^{3}$ and M. Willatzen ${ }^{2}$ \\ ${ }^{1}$ Institute of Technology and Innovation, University of Southern Denmark, DK-5230 Odense, Denmark \\ ${ }^{2}$ Department of Photonics Engineering, Technical University of Denmark, DK-2800 Kgs. Lyngby, Denmark \\ ${ }^{3}$ Department of Physics and Materials Science, City University of Hong Kong, Tat Chee Avenue, Kowloon Tong, Kowloon, Hong Kong
}

(Received 30 May 2013; revised manuscript received 26 July 2013; published 10 September 2013)

\begin{abstract}
We show that the acoustic response of perforated and elastically filled rigid screens can give rise to a broad landscape of tunable devices. We begin presenting deep-subwavelength transmission properties of a structured plate and demonstrate the immediate relationship to truly bound surface modes. We extend our theoretical model to analyze structured metal-fluid-metal wave guides for the confinement of sound and present exact expressions for the dispersion relations which describe the hybridization of resonances. We discuss the validity of our analytical model by direct comparison to full-wave simulations and use this technique in the search for broadband response in composite structures where the effective mass density and bulk modulus are simultaneously negative and exhibiting weak influences by viscous losses.
\end{abstract}

DOI: 10.1103/PhysRevB.88.100301

PACS number(s): 43.35.+d, 42.79.Dj, 81.05.Xj

Acoustic metamaterials with functionalities and properties designed by structural components in the form of resonating meta-atoms have been the subject of intense research in recent years because of their applications and potential use for the control of sound and elastic waves. It is difficult to generalize such a material in terms of its design and the mechanisms responsible for inducing the desired wave properties, as acoustic metamaterials have been created in many different ways. The first type of metamaterials designed was structures consisting of locally resonating building blocks in the form of rubber-coated metal spheres. ${ }^{1}$ When these inertial metamaterials are excited at the natural frequency, the core oscillates strongly out of phase with the driving force, giving rise to a negative mass density. Other types of metamaterials in the form of Helmholtz resonators and membranes have been employed and it thus seems that, to a large extent, design strategies have been inspired by microwave filter components. ${ }^{2-4}$ Recently, we also witness the emergence of perforated structures, which constitute flexible systems when designing broadband and all-angle negative refraction for sound. ${ }^{5-8}$

In this paper we investigate acoustic metadevices inspired by plasmonic building blocks and examine their feasibility in the design of sound confinement structures and tunable negative dispersion..$^{9-11}$ One example of a plasmonic building block is a perforated metal film, which has been the focus of extensive research activities associated with the phenomenon of extraordinary optical transmission (EOT). ${ }^{12}$ Since EOT was also observed in perfect conducting films not supporting the propagation of surface plasmons, it led to the consideration that a textured perfect conducting screen could sustain spoof surface EM modes very similar to plasmons in a bare metal film. In fact it has been shown that the effective permeability of those holey structures can be represented by a Drude expression dictated by the structure parameters. ${ }^{9}$ Recently, there has been much interest in related properties for the case of sound in structured and elastically filled screens. ${ }^{13-15} \mathrm{It}$ was shown that a Drude response can also be tailored in these materials and this fact motivates the present study to seek other plasmon-like behavior in acoustical systems. In this context we present an analytical study to describe coupled acoustoelastic surface waves in finite slabs and their link to transmission resonances. Furthermore, we build an analogy to an insulating layer sandwiched between two semi-infinite metals which can provide negative dispersion for surface plasmons. We demonstrate that a fluid layer between two structured half-spaces hosting negative mass densities sustain sound guiding behavior. Finally, we present results for doublenegativity $\left(\rho_{\text {eff }}<0\right.$ and $\left.1 / \kappa_{\text {eff }}<0\right)$ over an extended range of frequencies.

We begin by considering a structured half-space (taking $h \rightarrow \infty)$ as illustrated in the inset in Fig. 1. An elastic material such as rubber is clamped to a rigid immovable frame and arranged into a periodic lattice along the $x$ axis. The elastic material is treated as an isotropic solid, i.e., the displacements obey Navier's equations,

$$
\begin{gathered}
\rho \frac{\partial^{2} u_{x}}{\partial t^{2}}=\mu\left(\frac{\partial^{2} u_{x}}{\partial z^{2}}+\frac{\partial^{2} u_{x}}{\partial x^{2}}\right)+(\lambda+\mu)\left(\frac{\partial^{2} u_{x}}{\partial x^{2}}+\frac{\partial^{2} u_{z}}{\partial x \partial z}\right), \\
\rho \frac{\partial^{2} u_{z}}{\partial t^{2}}=\mu\left(\frac{\partial^{2} u_{z}}{\partial z^{2}}+\frac{\partial^{2} u_{z}}{\partial x^{2}}\right)+(\lambda+\mu)\left(\frac{\partial^{2} u_{z}}{\partial z^{2}}+\frac{\partial^{2} u_{x}}{\partial x \partial z}\right),
\end{gathered}
$$

where $\lambda, \mu$ and $\rho$ are the modulus of incompressibility (first Lamé coefficient), modulus of rigidity (second Lamé coefficient), and solid mass density, respectively. Due to translation invariance along the $y$ axis, we have $u_{y}=0$ and $\frac{\partial}{\partial y}=0$ everywhere. Since the walls around the rubber layer are rigid, we have

$$
\begin{aligned}
& u_{z}(x=0)=u_{z}\left(x=a_{x}\right)=0, \\
& u_{x}(x=0)=u_{x}\left(x=a_{x}\right)=0 .
\end{aligned}
$$

Further, at the fluid-rubber interface $(z=0)$, we must preserve continuity of the displacement $u_{z}$, the normal stress $\sigma_{z z}=$ $-p$, and, invoking the absence of shear stresses, $\sigma_{x z}=0$. This problem is not separable and exact analytical solutions do not exist. Hence, we can only attempt to find approximate analytical solutions. We do this by writing the ansatz

$$
u_{z}(x, z)=f_{z}(z) \cdot \sin \left(\frac{\pi}{a_{x}} x\right) e^{i \omega t}
$$




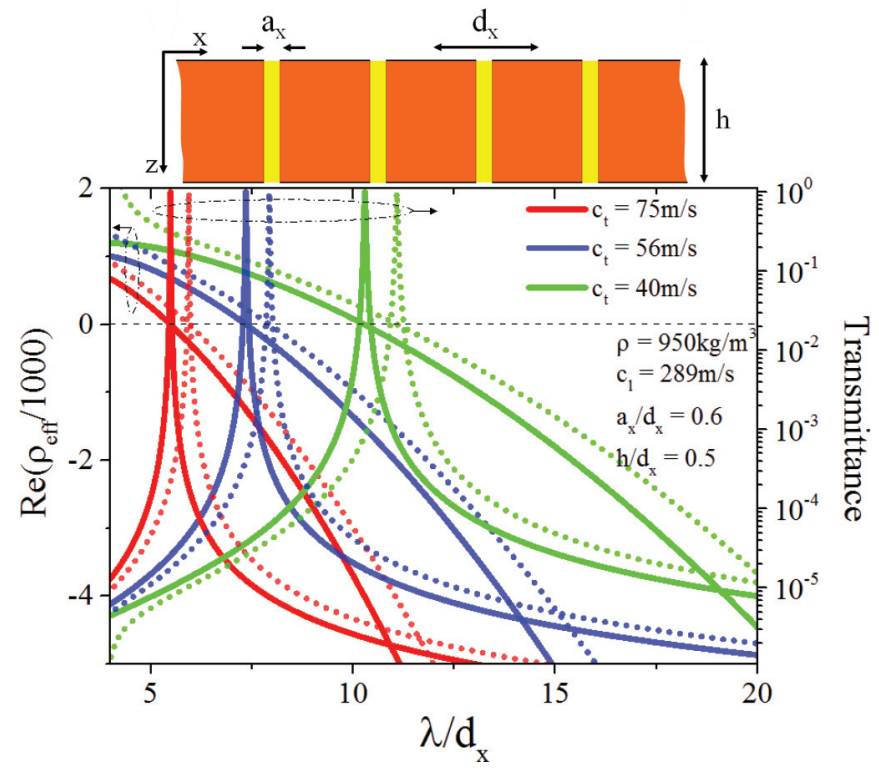

FIG. 1. (Color online) Transmittance and effective mass density spectra of the structured and elastically filled rigid screen surrounded by air. In all three cases the geometries are the same; we only vary $c_{t}$ as indicated. All calculations are undertaken for normally incident sound where the solid (dotted) lines represent data obtained by modal expansions (COMSOL simulation).

and assuming that $u_{z} \gg u_{x}$ such that the wave motion inside the elastic slit cavity predominantly resembles the transverse motion of a thin plate fixed at the edges, $x=0$ and $x=a_{x}$. To further justify this approximation, we have performed exact calculations using the finite-element method in COMSOL, verifying that indeed $u_{z} \gg u_{x}$. Note that, in Eq. (3), we have taken into consideration only the lowest order mode $(m=1)$, as it represents the field penetrating inside the cavity to a good approximation. Henceforth, neglecting a small contribution from $u_{x}$, an approximate solution is sought in the form

$$
f_{z}(z)=A \sin \left(\beta_{z} z+\phi\right),
$$

where $\phi$ is a phase, i.e.,

$$
u_{z}(x, z)=A \sin \left(\beta_{z} z+\phi\right) \cdot \sin \left(\frac{\pi}{a_{x}} x\right) e^{i \omega t} .
$$

In order to understand the wave nature of the displacement inside the elastic cavity we can solve for $\beta_{z}$ by substituting Eq. (5) into the second of Eqs. (1), which yields

$$
\beta_{z}=\omega \sqrt{\frac{\rho}{\lambda+2 \mu}} \sqrt{1-\frac{\mu}{\rho} \frac{\pi^{2}}{a_{x}^{2}} \frac{1}{\omega^{2}}}=\frac{\omega}{c_{l}} \sqrt{1-\frac{\omega_{p}^{2}}{\omega^{2}}} .
$$

From Eq. (6) we can see that a cutoff frequency locates at $\omega_{p}=\sqrt{\frac{\mu}{\rho}} \frac{\pi}{a_{x}}=c_{t} \frac{\pi}{a_{x}}$. This analytical finding is intriguing and in fact different from acoustic hole or slit arrays where the fundamental eigenmode is a constant $\beta_{z}=n_{h} k_{0}$.

In the following we derive an effective medium theory and modal expansions by rigorously coupling the acoustoelastic fields of the present structure. Plane-wave expansions are used in the reflection and transmission regions where the incident plane wave points along the $z$ axis. Inside the elastic cavities, the field is written as a linear combination of their eigenmodes.
However, as opposed to the approximate solution in Eq. (5), we consider the elastic material to be an anisotropic fluid. We can therefore write the $z$ components of the fields in that region as

$$
\begin{aligned}
u_{z} & =\sum_{m}\left(A_{m} e^{i \beta_{z}^{m} z}+B_{m} e^{-i \beta_{z}^{m} z}\right)\langle\mathbf{r} \mid m\rangle, \\
\sigma_{z z} & =i(\lambda+2 \mu) \sum_{m} \beta_{z}^{m}\left(A_{m} e^{i \beta_{z}^{m} z}-B_{m} e^{-i \beta_{z}^{m} z}\right)\langle\mathbf{r} \mid m\rangle,
\end{aligned}
$$

where $\beta_{z}^{m}=\frac{1}{c_{l}} \sqrt{\omega^{2}-m^{2} \omega_{p}^{2}}$ (with $m \geqslant 1$ ) and $\langle\mathbf{r} \mid m\rangle$ is the in-plane elastic field associated with mode $m$. As stated earlier, we impose continuity of the corresponding fields in order to arrive at linear expressions of unknown coefficients. We begin with a single interface $\left[B_{m}=0\right.$ in Eq. (7)] that accounts for an incident fluid-borne sound wave radiating onto a structured half-space. We can easily extract the specular reflection coefficient by taking the fundamental cavity eigenmode $(m=1)$ into account,

$$
R_{0}=\frac{Z_{\text {eff }} / Z_{0}-1}{Z_{\text {eff }} / Z_{0}+1}=\frac{\beta_{z}(\lambda+2 \mu)-\frac{\omega^{2} \rho_{0}}{k_{z}} \frac{8 a_{x}}{\pi^{2} d_{x}}}{\beta_{z}(\lambda+2 \mu)+\frac{\omega^{2} \rho_{0}}{k_{z}} \frac{8 a_{x}}{\pi^{2} d_{x}}},
$$

where $k_{z}=\sqrt{k_{0}^{2}-k_{x}^{2}}$ in free space. In other words, one can say that the incident acoustic wave is "seeing" a material of a given effective impedance $Z_{\text {eff }}=\rho_{\text {eff }} c_{\text {eff }}=\rho_{\text {eff }} \frac{\omega}{\beta_{z}}$, which can be derived from Eq. (8), when taking $k_{x}=0$. With the expression of $\beta_{z}$ in the metamaterial limit, Eq. (6), we can derive an effective fluidic mass density $\rho_{\mathrm{eff}}=\rho_{z}=Z_{\mathrm{eff}} \frac{\beta_{z}}{\omega}$ that is nothing but

$$
\rho_{\mathrm{eff}}=\frac{\pi^{2} d_{x} \rho}{8 a_{x}}\left(1-\frac{\omega_{p}^{2}}{\omega^{2}}\right) .
$$

In the following we wish to examine the scattering properties of structured slabs of finite thickness and demonstrate the immediate relationship to the effective mass density as presented in Eq. (9). By rigorously imposing continuity of the displacements and stresses at either side of the structured slab, that is, imposing continuity of the elastic cavity modes [Eq. (7) with $m=1$ ] to waves radiated, we arrive at a simple linear set of equations of unknown modal fields $\psi$ and $\psi^{\prime}$ :

$$
\left[\begin{array}{cc}
\left(G+\frac{i \beta_{z}(\lambda+2 \mu)}{\tan \left(\beta_{z} h\right)}\right) & \frac{i \beta_{z}(\lambda+2 \mu)}{\sin \left(\beta_{z} h\right)} \\
\frac{i \beta_{z}(\lambda+2 \mu)}{\sin \left(\beta_{z} h\right)} & \left(G+\frac{i \beta_{z}(\lambda+2 \mu)}{\tan \left(\beta_{z} h\right)}\right)
\end{array}\right]\left[\begin{array}{c}
\psi \\
\psi^{\prime}
\end{array}\right]=\left[\begin{array}{c}
2 \frac{\omega^{2} \rho_{0}}{k_{z}^{0}} S_{0} \\
0
\end{array}\right] .
$$

In (10) we write the wave vectors $k_{z}^{n}=\sqrt{k_{0}^{2}-\left(k_{x}^{n}\right)^{2}}$ with $k_{x}^{n}=$ $k_{x}^{0}+\frac{2 \pi}{d_{x}} n$ and the sum $G=\sum_{n} \frac{\omega^{2} \rho_{0}}{k_{z}^{n}}\left|S_{n}\right|^{2}$ with

$$
S_{n}=\sqrt{\frac{8}{a_{x} d_{x}}} \frac{\pi / a_{x}}{\left(k_{x}^{n}\right)^{2}-\pi^{2} / a_{x}^{2}} \cos \left(k_{x}^{n} \frac{a_{x}}{2}\right) .
$$

One can solve for the modal fields in Eq. (10) and then all scattering coefficients can be determined. ${ }^{16}$ In the metamaterial limit $\left(n=0\right.$ and $\left.k_{x} a_{x} \ll 1\right)$ we can reduce $S_{n}^{2}$ such that it only depends on geometrical parameters and we end up with a structure factor $S_{0}^{2}=S_{f}=\frac{8}{\pi^{2}} \frac{a_{x}}{d_{x}}$. Further to this, we apply the derived complex transmission $t_{n}=-\psi^{\prime} S_{n}$ and reflection $r_{n}=-\psi S_{n}+\delta_{0 n}$ coefficients to compute the 
effective acoustic parameters based on a retrieval technique. As rendered in Fig. 1 we plot the normal-incidence transmittance and the effective mass density $\rho_{\text {eff }}$ as a function of $\lambda / d x$ for a rigid plate containing a one-dimensional lattice of slits filled with a rubber material of various elastic parameters. The geometrical constants such as the slit width $a_{x}$ and thickness $h$ are held constant, while a rubber material of fixed solid mass density $\rho$ and longitudinal speed of sound $c_{l}$ is chosen as indicated in Fig. 1. By modifying the value of the transversal speed of sound $c_{t}$, as predicted by Eq. (9), we expect to spectrally control the location of the resonant transmission peak where the effective mass density $\rho_{\text {eff }}$ approaches 0 . Figure 1 illustrates three different cases where the lowering of $c_{t}$ results in the corresponding resonance frequency being lower as well. The spectral locations of full sound transmission do, in call cases, correspond to zero effective mass density, where $\omega=\omega_{p}$ as described by Eq. (9), the cutoff of a surface mode, which we explain later. Comparing the present technique with full-wave simulations (COMSOL), the overall trend of resonating frequencies is the same, although slightly shifted to long wavelengths as shown in Fig. 1.

In the following we demonstrate that the properties responsible for the resonant transmission peaks are well explained by the excitation of surface modes on and through the finite structure. For this to be true we are seeking truly bound modes by assuming waves to decay both into free space $\left(k_{z}=\right.$ $\left.i \sqrt{k_{x}^{2}-k_{0}^{2}}\right)$ and into the elastic inclusion $\left(\beta_{z}=\frac{i}{c_{l}} \sqrt{\omega_{p}^{2}-\omega^{2}}\right)$. We recall that a bare rigid plate without any structuring does not support surface-bound modes of this kind. ${ }^{16}$ One needs to pattern the structure either by perforation to support guided modes or by excitation of plate modes in nonrigid structures. The present theoretical formalism allows for the analytical expression of a dispersion relation (frequency vs $k_{x}$ ) of acoustic surface waves controlled by elastically filled screens. Imposing the above given conditions for bound modes we can express $k_{x}$ by the zero determinant in Eq. (10), which, in the deep-subwavelength limit $(n=0)$, is

$$
k_{x}=\frac{\omega}{c_{0}} \sqrt{1+\left(\frac{\rho_{0} c_{0}}{\rho c_{l}}\right)^{2} \frac{\omega^{2}}{\omega_{p}^{2}-\omega^{2}} S_{f}^{2} \gamma^{ \pm 2}}
$$

with $\gamma=\left(e^{-\frac{i \beta_{z} h}{2}}-e^{\frac{i \beta_{z} h}{2}}\right) /\left(e^{-\frac{i \beta_{z} h}{2}}+e^{\frac{i \beta_{z} h}{2}}\right)$, where the \pm sign determines the symmetry of the mode. In Fig. 2 we plot this dispersion relation, Eq. (12), with geometrical parameters as indicated in the caption. We predict a linear evolution of this function close to the sound line, but when approaching the hole cutoff (the acoustoelastic plasma frequency, $\omega=\omega_{p}$ ) we enter the flat and dispersionless regime at which sound decays evanescently away from the structured slab to which it is confined. Interestingly, at the cutoff frequency $\rho_{\mathrm{eff}}(\omega=$ $\left.\omega_{p}\right)=0$, which could lay the foundation for properties similar to those of the $\varepsilon$ near-zero materials in electromagnetism that are known to support tunneling and supercoupling-related phenomena. ${ }^{17,18}$ Also, in Fig. 2 we plot the transmittance versus normalized frequency and $k_{x}$ within (outside) the sound cone representing the excitation of leaky (bound) surface modes. When Bragg folding these coupled bound modes into the radiative part of the dispersion relation we exactly map the transmittance peaks. This connection is also given when

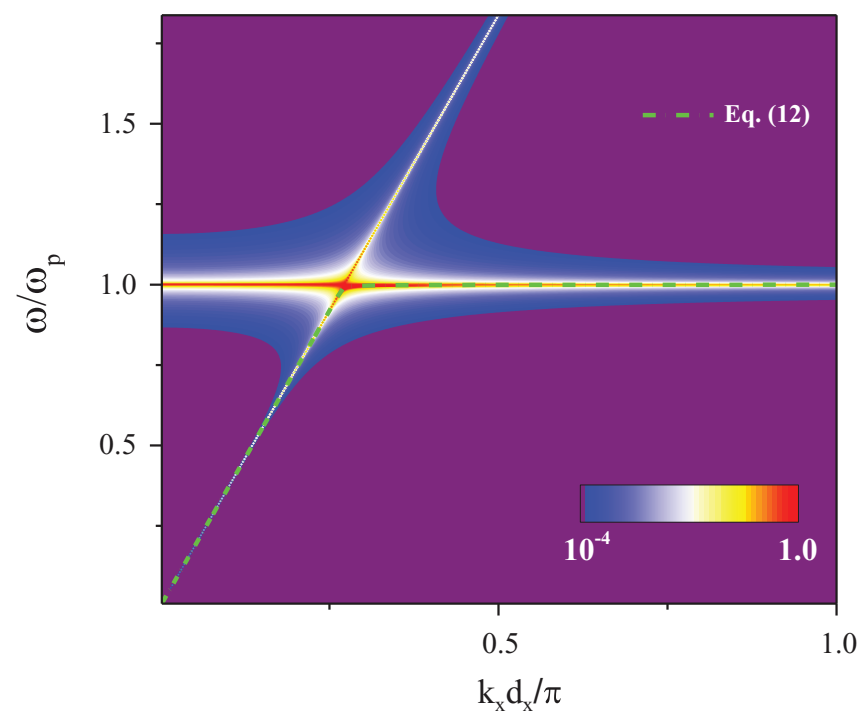

FIG. 2. (Color online) Analytical and numerical dispersion relations. The band diagram, transmittance as a function of $\omega$ and $k_{x}$, is mapped for a structure with the parameters $a_{x} / d_{x}=0.6$ and $h / d_{x}=0.5$ and contains filling parameters as in the latter example, though with $c_{t}=56 \mathrm{~m} / \mathrm{s}$. Additionally, we plot Eq. (12), the analytical dispersion relation for the bound coupled acoustoelastic surface mode.

plotting the transmittance for $k_{x}>k_{0}$ which coincides with the curve given by Eq. (12) plotted in Fig. 2.

Next, we wish to examine a configuration of two separated structured half-spaces which is an analog to an insulating film between two semi-infinite metals known to support guided surface plasmons for the case of light. Those structures are capable of supporting a negative index propagating mode with high figures of merit. ${ }^{10,11,19}$ These modes are not only tunable but can extend over a wide range of frequencies. In the present case we analyze its acoustical analogy by using the same theoretical formalism as presented above. Free-space scattering takes place in the region (air) separating the structured half-spaces (see the inset in Fig. 3), which is where sound is confined since waves decay evanescently into the structured slits by their fundamental cavity mode as long as $\omega_{p}>\omega$. We focus on the lowest possible guided mode within this regime, where we attempt to mimic the behavior of a fluid layer guided mode. Interestingly, we are able to create guided modes that decay away throughout the structure half-spaces whether we are within or outside the sound cone. In the deep-subwavelength regime, where the wavelength $\lambda$ is larger than all geometrical parameters, we can write the dispersion relation for these trapped modes,

$$
k_{x}=\frac{\omega}{c_{0}} \sqrt{1+\left(\frac{\rho_{0} c_{0}}{\rho c_{l}}\right)^{2} \frac{\omega^{2}}{\omega_{p}^{2}-\omega^{2}} S_{f}^{2} \gamma},
$$

where $\gamma=\tanh ^{2}\left(\sqrt{k_{x}^{2}-k_{0}^{2}} \frac{s}{2}\right)$ for $k_{x}>k_{0} \quad$ (propagating waves), $\quad \gamma=-\tan ^{2}\left(\sqrt{k_{0}^{2}-k_{x}^{2}} \frac{s}{2}\right)$ for $k_{0}>k_{x}$ (evanescent waves), and $s$ is the width of the gap. Note that the expression given by Eq. (13) is purely real whether we are outside or within the sound cone. We compare the exact dispersion relation from Eq. (13) with full-wave simulations (COMSOL) 

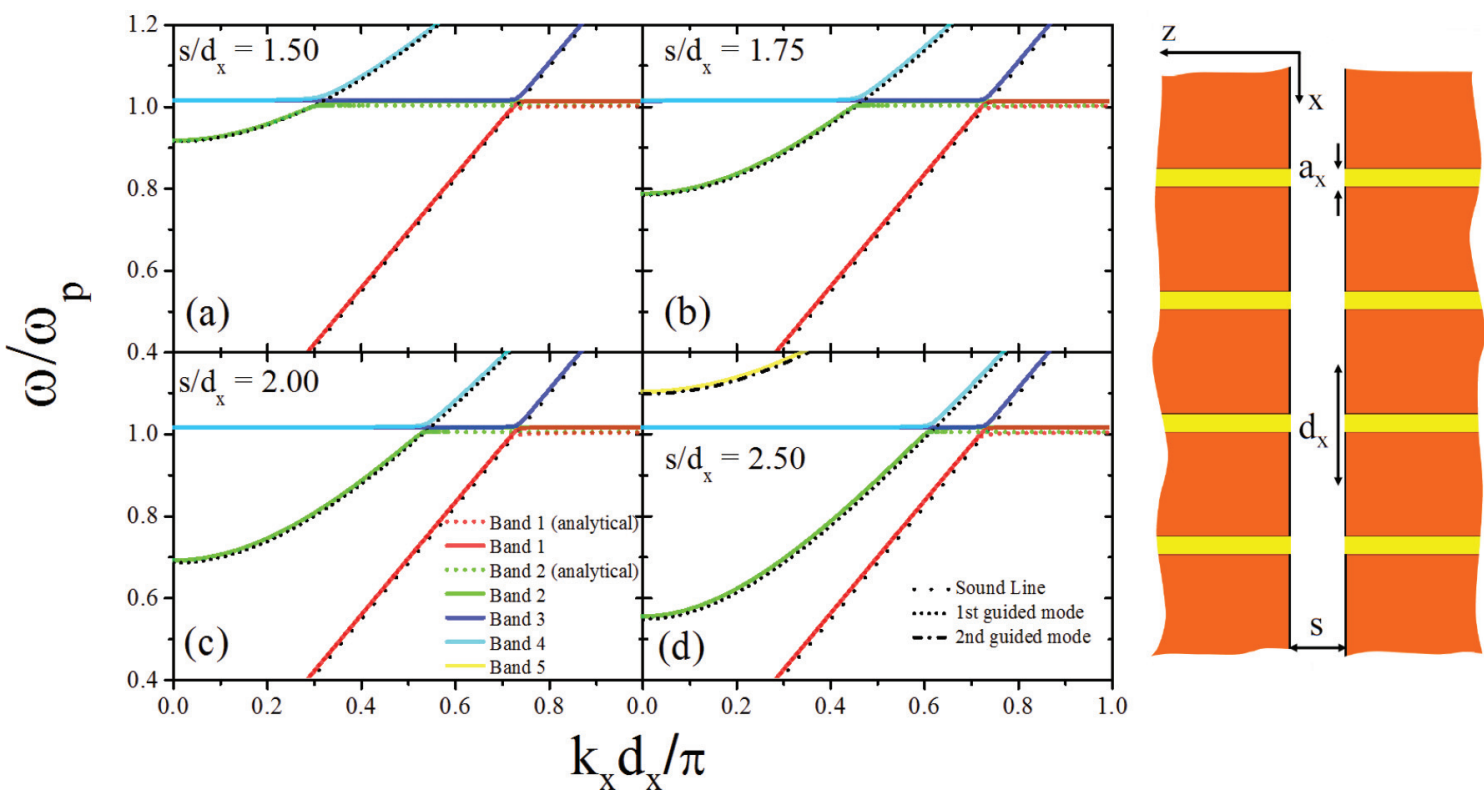

FIG. 3. (Color online) Dispersion relation of structured metal-fluid-metal wave guides. An air layer of thickness $s$ separates two half-spaces structured by slits of width $a_{x} / d_{x}=0.6$ and filled by an elastic material with $\rho=950 \mathrm{~kg} / \mathrm{m}^{3}, c_{l}=289 \mathrm{~m} / \mathrm{s}$, and $c_{t}=150 \mathrm{~m} / \mathrm{s}$. The confined modes are plotted for four values of the separation $s$ as indicated in each panel.

where all material and geometrical parameters apart from the gap separation $s$ are held constant as indicated in Fig. 3. In all plotted cases we predict a strong resonance dependence with the gap separation $s$. The nature of the bands can be understood by the odd and even spoof surface modes (induced by the structured half-spaces) hybridizing with the zeroth-order sound line and the first-order fluid guided mode, respectively [see Figs. 3(a)-3(c)]. In Fig. 3(d) we see a similar behavior, but due to a larger gap separation $s / d_{x}=2.50$, we are able to excite higher ordered modes in the plotted range. While the upper edge of the spoof surface modes for all simulated cases asymptotically approaches the limit $\omega=\omega_{p}$, the lower edge always coincides with a $\lambda$ half-resonance, $s=\lambda / 2$. When we enter into the spectrally narrow and flat regime around $\omega_{p}$, it is possible to trap sound with the advantage of providing omnidirectional guiding. It is also shown that with an enlargement of $s$ we can broaden the entire bandwidth of the corresponding mode, as the lower edge consequently moves towards lower frequencies. This in turn has the fortunate advantage of leading to stronger confinement within the gap as a consequence of shorter penetration into the structured regions, $\omega \ll \omega_{p}$.

Throughout this work we have analyzed the complex interaction between fluid-borne sound and resonators in the form of elastic cavities. We have treated the elastic material as an anisotropic fluid and learned that this assumption holds fairly well when making comparisons to full-wave simulations as shown in Figs. 1 and 3. Naturally, when imposing these assumptions one needs to deal with limitations, e.g., we took the in-plane cavity motion to be sinusoidal [Eq. (3)], but for very thin structures it could as well have been a bending-type motion. In fact we show by full-wave simulations (COMSOL) that the choice of thin plates ( $h=0.25 d_{x}$, for example) can lead to broader resonances. As an example, we take a composite metamaterial consisting of two facing structured rigid plates that host resonances with a negative index caused by double-negativity behavior. ${ }^{15}$ As shown in Fig. 4, we obtain an extended pass band of full transmission which is the broad region where both the effective mass density $\rho_{\text {eff }}$ and the bulk modulus $1 / \kappa_{\text {eff }}$ are negative. By the specific choice of a very thin structure, we believe that the excitation of bending modes inside the cavities leads to the expected phenomena of negative

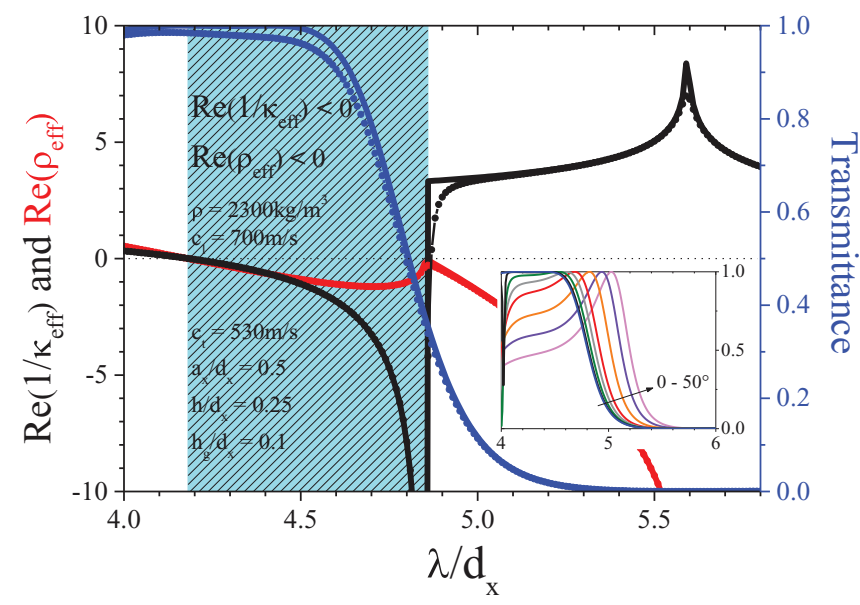

FIG. 4. (Color online) Broadband double-negativity. A composite metamaterial consisting of two separated structured and elastically filled plates is immersed in water and tuned for maximal broadband response with negative effective mass density $\rho_{\text {eff }}<0$ and bulk modulus $1 / \kappa_{\text {eff }}<0$ (shaded region). The structure and filling parameters are as indicated and inside the gap we take a fluid with $\rho_{g} / \rho_{0}=1$ and $c_{g} / c_{0}=0.27$. Dotted lines in the spectrum represent data obtained in the presence of viscous losses. Inset: Transmittance spectrum for various angles in the range $0-50^{\circ}$. 
index behavior, however, with an extended bandwidth. The dotted lines in Fig. 4 represent calculations of the transmittance and the effective parameters comprising realistic viscous losses in the fluid regions. ${ }^{7}$ Interestingly and fortunately, the influence of losses is marginal and the same can be said for the angular response as shown in the inset in Fig. 4. Supporting broadband behavior for many angles of incidence together with the insensitivity to viscous losses proves the robustness of this double-negativity scheme.

In summary, we have demonstrated that an acoustic surface wave equivalent to spoof surface plasmons can be engineered in rigid structures when they are pierced with holes and filled with an elastic inclusion. These surface waves, when designed for finite plates, are responsible for transmission resonances through tiny holes and can likewise be guided between two facing structured half-spaces. These new types of acoustic metadevices can thus be tuned to produce broadband resonances or omnidirectional propagation for a narrow selective range of frequencies useful for many interesting sound control applications.

All authors acknowledge Jensen Li for stimulating discussions. J.C. gratefully acknowledges financial support from the Danish Council for Independent Research and a Sapere Aude grant (No. 12-134776). Z.L. thanks the GRO program of Samsung Advanced Institute of Technology for support. *jochri@fotonik.dtu.dk

${ }^{1}$ Z. Liu, X. Zhang, Y. Mao, Y. Y. Zhu, Z. Yang, C. T. Chan, and P. Sheng, Science 289, 1734 (2000).

${ }^{2}$ N. Fang, D. Xi, J. Xu, M. Ambati, W. Srituravanich, C. Sun, and X. Zhang, Nat. Mater. 5, 452 (2006).

${ }^{3}$ S. H. Lee, C. M. Park, Y. M. Seo, Z. G. Wang, and C. K. Kim, J. Phys.: Condens. Matter 21, 175704 (2009).

${ }^{4}$ S. H. Lee, C. M. Park, Y. M. Seo, Z. G. Wang, and C. K. Kim, Phys. Rev. Lett. 104, 054301 (2010).

${ }^{5}$ J. Christensen and F. J. Garcia de Abajo, Phys. Rev. Lett. 108, 124301 (2012).

${ }^{6}$ J. Christensen and F. J. Garcia de Abajo, Phys. Rev. B 86, 024301 (2012).

${ }^{7}$ Z. Liang, T. Feng, S. Lok, F. Liu, K. B. Ng, C. H. Chan, J. Wang, S. Han, S. Lee, and J. Li, Sci. Rep. 3, 1614 (2013).

${ }^{8}$ Y. Xie, B.-I. Popa, L. Zigoneanu, and S. A. Cummer, Phys. Rev. Lett. 110, 175501 (2013).
${ }^{9}$ J. B. Pendry, L. Martin-Moreno, and F. J. Garcia-Vidal, Science 305, 847 (2004).

${ }^{10}$ H. Shin and S. Fan, Phys. Rev. Lett. 96, 073907 (2006).

${ }^{11}$ H. J. Lezec, J. A. Dionne, and H. A. Atwater, Science 316, 430 (2007).

${ }^{12}$ T. W. Ebbesen, H. J. Lezec, H. F. Ghaemi, T. Thio, and P. A. Wolff, Nature 391, 667 (1998).

${ }^{13}$ S. Yao, X. Zhou, and G. Hu, New J. Phys. 12, 103025 (2010).

${ }^{14}$ R. Hao, C. Qiu, Y. Ye, C. Li, H. Jia, M. Ke, and Z. Liu, Appl. Phys. Lett. 101, 021910 (2012).

${ }^{15}$ Z. Liang, M. Willatzen, J. Li, and J. Christensen, Sci. Rep. 2, 859 (2012).

${ }^{16}$ J. Christensen, L. Martin-Moreno, and F. J. Garcia-Vidal, Phys. Rev. Lett. 101, 014301 (2008).

${ }^{17}$ M. Silveirinha and N. Engheta, Phys. Rev. Lett. 97, 157403 (2006).

${ }^{18}$ R. Fleury and A. Alú, Phys. Rev. Lett. 111, 055501 (2013).

${ }^{19}$ J. A. Dionne, E. Verhagen, A. Polman, and H. A. Atwater, Opt. Express. 16, 19001 (2008). 